\title{
Evaluación de la restauración de la diversidad fúngica en un área reforestada con Polylepis australis (Rosaceae): un estudio de caso
}

\author{
Ana L. Gallo ${ }^{1, \varpi}$; Gerardo Robledo ${ }^{1} ;$ Marcos Landi $^{2} \&$ Carlos Urcelay ${ }^{1}$ \\ 1 Universidad Nacional de Córdoba, CONICET, Instituto Multidisciplinario de Biología Vegetal, Laboratorio de Micología. \\ Córdoba, Argentina. 2 Universidad Nacional de Córdoba, Facultad de Ciencias Exactas, Físicas y Naturales, Cátedra de \\ Ecología General. Córdoba, Argentina.
}

\begin{abstract}
Resumen. Los bosques de Polylepis australis han sido muy disturbados y la reforestación es una alternativa para restaurar esos ecosistemas. El objetivo del presente estudio es evaluar la restauración de la comunidad fúngica en un bosque de $P$. australis reforestado. Nuestra hipótesis de trabajo es que la comunidad fúngica se modifica en concordancia con las situaciones del bosque: alta degradación, bosque natural maduro y bosque restaurado. Se compararon variables fúngicas (riqueza, abundancia y equitatividad de macromicetes) de un área nativa, de un área degradada y reforestada y de un área degradada sin reforestación. Para ello se establecieron 15 parcelas de $5 \times 5 \mathrm{~m}$ y se muestrearon cinco por estación. Se recolectaron las fructificaciones de macromicetes, y luego se las clasificó en grupos morfo-taxonómicos. Se hicieron análisis de componentes principales y de correlación entre las variables fúngicas y la estructura vegetal, y análisis de correlación entre las variables fúngicas y las variables químicas del suelo. La riqueza, la abundancia y la equitatividad fueron significativamente mayores en el área nativa, intermedias en el área reforestada y menores en el área degradada. Las parcelas del área nativa se caracterizaron por una alta cobertura de árboles maduros, las del área reforestada por graminoides y árboles jóvenes y las del área degradada por herbáceas latifoliadas. Las tres variables fúngicas fueron máximas a mayor cobertura arbórea y correlacionaron positivamente con el fósforo y $\mathrm{pH}$ del suelo. Los resultados muestran que 12 años después de que un área degradada fuera reforestada con P. australis, la diversidad fúngica es mayor que la de un área degradada, pero está lejos de alcanzar los niveles del área nativa. Se postula que los hongos serían bioindicadores sensibles a la degradación y la restauración de los bosques.
\end{abstract}

[Palabras clave: hongos, macromicetes, riqueza, abundancia, equitatividad, reforestación, bioindicadores, Argentina]

\begin{abstract}
Aвstract. Evaluation of fungal diversity restoration in an afforestation of Polylepis australis (Rosaceae): a case study: Polylepis australis forest had been significantly disturbed and afforestation is an alternative to restore these ecosystems. The aim of this study is to evaluate the restoration of the fungal community in P. australis afforestation. Our hypothesis is that the fungal community varies between forest situations: high degraded, mature forest, and restored forest. Fungal variables (richness, abundance and evenness of macromicetes) were compared between a native area, an afforestation and a degraded land. To this end, 15 plots of $5 \times 5 \mathrm{~m}$ were established and five of them were sampled in each season. Macromicetes fruiting bodies were collected and classified into different morpho-taxonomical groups. Principal Components Analyses and correlation analyses between the fungal variables and the plot's structure were performed as well as correlation analysis between the fungal variables and soil chemical variables. Richness, abundance and evenness were significantly higher in the native area, intermediate in the afforestation and lower in the degraded land. The native area's plots showed high tree cover, the afforestation's plots high graminoid and young trees cover, and the degraded land's plots high forbs cover. Values of fungal variables were higher at higher tree cover and were positively correlated with soil phosphorus content and $\mathrm{pH}$. The results show that, after 12 years of afforestation with $P$. australis, the fungal diversity in that ecosystem is higher than the observed in reference degraded land but is far from the observed in a native area. It can be hypothesized that fungi are sensitive bioindicators of forest degradation and restoration.
\end{abstract}

[Keywords: fungi, macromicetes, richness, abundance, evenness, afforestation, bioindicators, Argentina]

\section{INTRODUCCIÓN}

La mayoría de las áreas boscosas de nuestro planeta están siendo sometidas a procesos de fragmentación, degradación y cambios en la biodiversidad, que influyen en el

\section{Editora asociada: Adriana Ruggiero}

lgallo@correociefap.org.ar funcionamiento de dichos ecosistemas. Esta problemática puede ser afrontada mediante la disminución de las tasas de pérdida de bosques y mitigada a través de procesos de restauración de los ecosistemas (Hobbs 2007; Chazdon 2008). 
La "restauración ecológica" es el proceso mediante el cual se asiste a la recuperación de un ecosistema que ha sido dañado, degradado o destruido (SER 2002), y cuyo objetivo principal es restablecer las funciones, servicios y resiliencia de dicho ecosistema (Chapman \& Underwood 2000). En el caso de los bosques, cabe destacar que si bien la restauración puede recuperar muchas funciones ecosistémicas y muchos componentes de la biodiversidad original, el bosque que emerja en lugares con impacto humano nunca será igual, en composición de especies, al bosque antiguo (Hobbs et al. 2006).

Los bosques del género Polylepis (Rosaceae) se distribuyen a lo largo de los andes tropicales desde Venezuela hasta el centro de Argentina (Fjeldsa \& Kessler 1996). Polylepis australis Bitter es la única especie extra-andina que alcanza el límite de distribución austral del género en las montañas pampeanas del centro de Argentina. Los bosques de P. australis albergan numerosos endemismos de flora y micota (Cabido et al. 2003; Robledo et al. 2006; Hernández Caffot et al. 2013; Renison et al. 2013 y referencias allí citadas). Sumado a ello, brindan servicios ecosistémicos importantes (e.g., provisión de agua) ya que representan las comunidades vegetales principales que protegen las cabeceras de cuencas de las Sierras Grandes de Córdoba.

Sin embargo, el estado de conservación de los bosques de $P$. australis es variable a lo largo de su distribución (Renison et al. 2013). Estos bosques han sido históricamente sometidos a cría de ganado, fuego y extracción de madera. Como consecuencia, han quedado restringidos a quebradas y rodeados de diferentes tipos de pastizales (Cingolani et al. 2004; Renison et al. 2006); en lugares más expuestos se transformaron en bosques abiertos y compuestos de árboles más bajos (Enrico et al. 2004). Si bien se supone que cubrieron áreas más amplias en el pasado (Renison et al. 2006; Cingolani et al. 2008), en la actualidad ocupan apenas $12 \%$ de la cadena montañosa. Sólo $2.5 \%$ corresponde a bosque cerrado, con especies leñosas exóticas (Renison et al. 2013). Los cambios causados en la vegetación alteran las condiciones microclimáticas, lo cual afecta de manera negativa la recuperación natural del bosque (Torres et al. 2008). Por esta razón, la reforestación quizás sea la única opción para amortiguar los cambios en el ecosistema, al menos en el corto y mediano plazo. Con este fin, en el año 1997 comenzó una reforestación con P. australis en las Sierras Grandes de
Córdoba, que continúa hasta la actualidad.

La forma ideal de plantear un estudio que permita evaluar la eficiencia de la reforestación en la restauración de un ecosistema es mediante la comparación con registros de sitios sin restaurar y de bosques remanentes (Chapman \& Underwood 2000; Wilkins et al. 2003). Este abordaje requiere del estudio de diversos grupos de organismos biológicos, principalmente aquellos implicados en procesos ecosistémicos esenciales en el funcionamiento de los bosques. Si bien esto es ampliamente reconocido, la mayoría de las evaluaciones de reforestaciones con especies nativas se centran en plantas y animales, mientras que otros grupos biológicos han sido poco estudiados. Tal es el caso de los hongos, con excepción de los micorrícicos (Ruiz-Jaen \& Aide 2005).

Los hongos son organismos heterótrofos clave en el ciclado de nutrientes en los ecosistemas terrestres (Cromack Jr. \& Caldwell 1992). La composición de las comunidades fúngicas se encuentra vinculada de forma estrecha con la disponibilidad de recursos, lo que se relaciona, a su vez, con la estructura vegetal del lugar (Ponge 2005). Además, se ha observado que los cambios en la composición de las comunidades fúngicas influyen en el funcionamiento de los ecosistemas (Swift 2005). Si bien existen trabajos en los que se estudian las comunidades fúngicas de bosques en distintos estadios sucesionales, sujetos a diferentes tipos de manejo forestal y forestaciones en campos de cultivo o forestaciones con especies exóticas (Bader et al. 1995; Lindner et al. 2006; Kujawa \& Kujawa 2008; Oria de Rueda et al. 2010; Robledo \& Renison 2010), no hay estudios que evalúen las comunidades de macromicetes (a través del registro de sus esporomas) en respuesta a procesos de reforestación con especies nativas.

En este trabajo nos propusimos evaluar la restauración de un área reforestada con $P$. australis de 12 años de antigüedad, en relación a las comunidades fúngicas (macromicetes), tomando como puntos de comparación un área nativa de bosque maduro remanente y un área degradada similar a la situación prereforestación. Dado que la reforestación con dichas características es única en el rango de distribución de la especie, no se encuentran repeticiones en el paisaje. Por esta razón, el presente constituye un estudio de caso en un marco descriptivo y el alcance de los resultados se limita a ello. 
En particular, comparamos las variables fúngicas (riqueza, abundancia y equitatividad) de las comunidades de macromicetes entre las tres áreas mencionadas, en verano, otoño y primavera. Además, examinamos las posibles relaciones entre las variables fúngicas y la estructura vegetal y las variables químicas del suelo.

\section{MATERIALES Y MÉTODOS}

\section{Área de estudio}

El estudio se llevó a cabo en el macizo de "Los Gigantes", sector norte de las Sierras Grandes de Córdoba (Argentina). Las Sierras Grandes son una cadena montañosa que se extiende entre 1700 y 2800 m.s.n.m. y representan una isla biogeográfica con bosques de $P$. australis y numerosos endemismos (Cabido et al. 1998). A los 2100 m.s.n.m., las temperaturas medias de los meses más fríos y más cálidos son 5 y $11.4{ }^{\circ} \mathrm{C}$, respectivamente, sin período libre de heladas (Cabido 1985). La precipitación media anual es $920 \mathrm{~mm}$, con el 80\% de las precipitaciones concentrado en los meses cálidos.

Los sitios de estudio fueron elegidos por poseer diferentes estados de conservación: a) bosque natural maduro de $P$. australis o área nativa (AN) ubicadoen "LaQuebrada de Anselmo" (31 23'42.31" S y $\left.64^{\circ} 49^{\prime} 4.93^{\prime \prime} \mathrm{W}\right)$, b) área reforestada (AR) con $P$. australis de 12 años de antigüedad que se encuentra dentro de una clausura correspondiente al Proyecto de Reforestación $\left(31^{\circ} 25^{\prime} 4.39^{\prime \prime}\right.$ S y $64^{\circ} 48^{\prime} 22.08^{\prime \prime}$ W), y c) un área degradada (AD) localizada en "El valle de Cuello" (31 $25^{\prime} 3.31^{\prime \prime}$ S y $64^{\circ} 48^{\prime} 36.72^{\prime \prime}$ W). Previo a la reforestación, el AR presentaba una situación similar, incluyendo la misma historia de uso que el
AD. Ambas se encuentran a la misma altitud, 2200 m.s.n.m. y media una distancia de $400 \mathrm{~m}$ entre ellas. El AN se encuentra a $2.4 \mathrm{~km}$ de las otras áreas, a una altitud menor (1900 m.s.n.m.). El hecho de haber elegido este sitio a pesar de esas diferencias se debe a que el AN constituye el parche de P. australis accesible más próximo y similar en términos de topografía a las AR y AD.

En cada área (AN, AR y AD) se establecieron 15 parcelas de $5 \times 5 \mathrm{~m}$ y se muestrearon cinco parcelas diferentes en cada estación (verano, otoño y primavera) ya que, si bien la mayoría de los macromicetes fructifican en otoño, también existen registros de hongos que fructifican en primavera y/o verano (Moore et al. 2008). En cada sitio las parcelas fueron establecidas y muestreadas al azar.

\section{Recolección y clasificación de macromicetes}

Se recolectaron los esporomas - cuerpos de fructificación de los macromicetes- presentes (hongos de los Phyla Ascomycota y Basidiomycota que forman esporomas macroscópicos). Cada una de las colecciones fue asignada a un grupo morfotaxonómico. Dichos grupos fueron: discomicetes, "otros ascomicetes" (Phylum Ascomycota), agaricoides, corticioides, gelatinosos, gasteroides y poliporoides (Phylum Basidiomycota). Luego, mediante observaciones microscópicas, se los identificó a nivel de género o especie. En aquellos casos en que no fue posible determinar el género, la colección fue nombrada de acuerdo al grupo morfo-taxonómico en el que había sido incluido. Las colecciones fueron depositadas en el herbario CORD (Museo Botánico, Facultad de Ciencias Exactas, Físicas y Naturales, Universidad Nacional de Córdoba).

Tabla 1. Cobertura (\%) de las parcelas en las tres áreas de estudio (área nativa, área reforestada, área degradada).

Table 1. Plot's cover (\%) in the three study areas (native area, afforestation, disturbed area).

\begin{tabular}{|c|c|c|c|c|c|c|c|c|c|}
\hline & \multicolumn{3}{|c|}{ Área nativa } & \multicolumn{3}{|c|}{ Área reforestada } & \multicolumn{3}{|c|}{ Área degradada } \\
\hline & 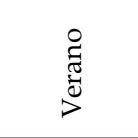 & 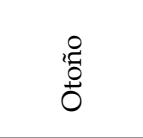 & $\begin{array}{l}\widetilde{\Xi} \\
\stackrel{\Xi}{\Xi} \\
\stackrel{\Xi}{\Xi}\end{array}$ & $\begin{array}{l}\stackrel{0}{0} \\
\text { गूँ } \\
>\end{array}$ & $\begin{array}{l}\stackrel{0}{0} \\
\text { J゙ }\end{array}$ & 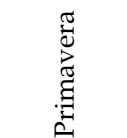 & $\begin{array}{l}\stackrel{0}{0} \\
\stackrel{0}{0} \\
>\end{array}$ & 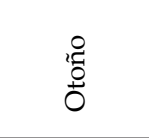 & 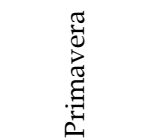 \\
\hline Árboles & $67.0(19.0)$ & $42.0(7.2)$ & $21.0(3.3)$ & 33.4 (11.6) & $34.4(16.0)$ & $24.0(4.9)$ & 0 & 0 & 0 \\
\hline Arbustos & $4.0(1.9)$ & $3.8(2.8)$ & $19.0(4.3)$ & $1.8(1.4)$ & $5.4(2.0)$ & $0.4(0.4)$ & 0 & $0.8(0.6)$ & $0.6(0.6)$ \\
\hline $\begin{array}{l}\text { Herbáceas } \\
\text { latifoliadas }\end{array}$ & $6.0(1.7)$ & $1.6(0.4)$ & $8.4(1.0)$ & $27.4(7.2)$ & $20.2(5.5)$ & $12.4(6.3)$ & $73.6(6.7)$ & $27.0(5.9)$ & $38.2(6.8)$ \\
\hline Graminoides & $13.4(6.3)$ & $46.6(15.1)$ & $35.0(10.4)$ & $24.6(4.9)$ & $58.0(5.7)$ & $60.0(3.5)$ & $19.2(5.5)$ & $40.4(5.3)$ & $27.6(9.6)$ \\
\hline Helechos & $50.2(13.9)$ & $11.6(6.3)$ & $3.6(1.1)$ & $3.0(1.8)$ & $1.0(1.0)$ & $1.2(1.0)$ & 0 & $0.4(0.4)$ & $0.6(0.4)$ \\
\hline Briófitas & 0 & $1.2(1)$ & $2.40(0.9)$ & $0.6(0.4)$ & $0.8(0.4)$ & $0.6(0.4)$ & $1.4(1.0)$ & 0 & $1.4(0.8)$ \\
\hline Mantillo & $12.6(4.0)$ & $11.8(3.6)$ & $11.6(4.8)$ & $16.0(9.8)$ & $5.6(3.7)$ & $9.4(5.5)$ & 0 & 0 & 0 \\
\hline Suelo desnudo & $2.4(1.3)$ & $2.2(0.8)$ & $4.20(1.6)$ & $1.8(0.9)$ & $1.0(0.5)$ & $7.0(7.0)$ & $4.8(1.5)$ & $6.8(3.4)$ & $5.6(3.7)$ \\
\hline Roca & $15.4(7.7)$ & $23.8(11.8)$ & $25.0(8.7)$ & $16.2(11.3)$ & $13.4(4.9)$ & $7.6(4.2)$ & $1.0(1.0)$ & $22.0(10.2)$ & $22.0(13.8)$ \\
\hline
\end{tabular}


Tabla 2. Nutrientes del suelo en las tres áreas de estudio (área nativa, área reforestada, área degradada).

Table 2. Soil nutrients in the three study areas (native area, afforestation, disturbed area).

\begin{tabular}{|c|c|c|c|c|c|c|c|c|c|}
\hline & & rea nativ & & & a refores & ada & & ea degra & ada \\
\hline & Verano & Otoño & Primavera & Verano & Otoño & Primavera & Verano & Otoño & Primavera \\
\hline Materia & 12.1 & 11.8 & 8.6 & 9.5 & 11.2 & 11.4 & 10.9 & 6.9 & 11.4 \\
\hline & (2.6) & (1.5) & $(0.7)$ & (2.6) & (2.4) & (2.5) & $(2.4)$ & $(0.8)$ & (1.9) \\
\hline Nitrógeno & 0.5 & 0.5 & 0.4 & 0.4 & 0.5 & 0.6 & 0.5 & 0.3 & 0.6 \\
\hline total (\%) & $(0.1)$ & $(0.1)$ & $(0.0)$ & $(0.1)$ & $(0.1)$ & $(0.1)$ & $(0.1)$ & $(0.0)$ & $(0.1)$ \\
\hline $\mathrm{C} / \mathrm{N}$ & 13.7 & 13.3 & 11.8 & 15.0 & 12.7 & 11.8 & 13.3 & 12.4 & 12.1 \\
\hline & $(1.2)$ & $(0.6)$ & $(0.2)$ & $(2.7)$ & $(0.7)$ & $(0.2)$ & $(0.7)$ & $(0.4)$ & $(0.3)$ \\
\hline Nitrato & 64.6 & 34.4 & 4.2 & 31.6 & 22.6 & 6.8 & 13.2 & 8.8 & 4.2 \\
\hline (ppm) & $(30.4)$ & $(22.4)$ & $(0.6)$ & $(16.5)$ & (3.8) & (2.6) & $(2.4)$ & (1.6) & $(1.8)$ \\
\hline Amonio & 24.9 & 9.1 & 1.9 & 11.5 & 10.8 & 2.7 & 5.5 & 4.5 & 1.6 \\
\hline (ppm) & (9.3) & (3.7) & $(0.2)$ & (5.3) & (2.1) & $(0.8)$ & (1.2) & $(0.7)$ & $(0.6)$ \\
\hline Fósforo & 24.7 & 14.2 & 9.8 & 10.6 & 6.1 & 7.4 & 7.8 & 4.3 & 3.6 \\
\hline (ppm) & (5.7) & (5.0) & $(1.2)$ & (3.0) & $(0.9)$ & (2.1) & (2.2) & $(1.8)$ & (1.1) \\
\hline $\mathrm{pH}$ & 6.5 & 5.2 & 4.9 & 5.1 & 4.7 & 5.0 & 5.1 & 4.8 & 4.8 \\
\hline & (0.3) & $(0.1)$ & $(0.1)$ & $(0.2)$ & $(0.1)$ & (0.3) & $(0.1)$ & $(0.1)$ & $(0.1)$ \\
\hline
\end{tabular}

Determinación de la estructura de la vegetación $y$ de las variables químicas del suelo

En cada parcela se determinó la estructura de la vegetación mediante la estimación visual de la cobertura de distintas formas de vida (Kent \& Coker 1992). El porcentaje de cobertura fue establecido para árboles, arbustos, herbáceas latifoliadas, graminoides, helechos, briófitas, mantillo, suelo desnudo y roca (Tabla 1). Además, se contaron las unidades de guano (von Müller et al. 2012).

Para determinar las variables químicas del suelo se tomaron 45 muestras de suelo de $10 \mathrm{~cm}$ de profundidad; 15 muestras (una por parcela) correspondieron a cada situación por cada estación (Tabla 2). Los métodos utilizados fueron Walkley \& Black para materia orgánica (\%) y carbono orgánico (\%), Kjeldahl para nitrógeno total (\%), potenciómetro directo para nitrato y amonio (ppm), Bray \& Kurtz No. 1 para fósforo (ppm) y $10 \mathrm{~g}$ de suelo en peso seco mezclado con agua destilada para $\mathrm{pH}$ (Sparks et al. 1996). Los análisis se realizaron en el laboratorio de Edafología de la Facultad de Ciencias Agropecuarias (Universidad Nacional de Córdoba, Argentina).

\section{Análisis de datos}

Se describió la composición de especies y se analizaron la riqueza, abundancia y equitatividad de macromicetes. Estas variables fueron determinadas a partir del registro de sus esporomas. Para estimar el esfuerzo de muestro de especies en cada situación se calcularon curvas de rarefacción con el programa EstimateS (Colwell 2009) a partir del número de colecciones de cada especie por parcela. Para realizar una estimación sobre el nivel de representatividad de las comunidades de hongos obtenida con los muestreos llevados a cabo en cada situación, se calculó el estimador de riqueza de especies mínima Chao2 (Unterseher et al. 2008).

Para determinar si existen diferencias entre las variables fúngicas en las distintas áreas en cada estación se realizó un ANOVA de dos factores. Debido a que las áreas no siempre se encontraron exactamente a la misma altitud, se incluyó la altura sobre el nivel del mar como covariable, pero no fue significativa. Dado que las variables no cumplieron con los supuestos de homogeneidad de la varianza y/o normalidad de los errores, los datos fueron transformados a rangos (Zar 1999). Se utilizó el test de Fisher "a posteriori" para determinar las diferencias entre las situaciones. Además, se realizó un análisis de componentes principales (ACP), y se empleó como criterio de clasificación a la parcela en función de los datos de estructura vegetal de cada parcela. Luego se realizaron análisis de correlación de Spearman entre las variables fúngicas y los dos primeros ejes del ACP (estructura de la vegetación) y con cada una de las variables químicas del suelo. Para los análisis se utilizó el paquete estadístico Infostat versión 2013 (Di Rienzo et al. 2013).

\section{Resultados}

Se encontraron 286 esporomas, correspondientes a 75 especies de 
Tabla 3. Macromicetes en las tres áreas de estudio (área nativa, área reforestada, área degradada) y en las tres estaciones (verano, otoño, primavera).

Table 3. Macromicetes in the three study areas (native area, afforestation, disturbed area) and in the three seasons (summer, autumn, spring).

\begin{tabular}{|c|c|c|c|c|c|c|c|c|c|c|}
\hline \multirow{2}{*}{$\begin{array}{l}\text { Grupo morfo } \\
\text { taxonómico }\end{array}$} & \multirow[t]{2}{*}{ Macromicetes } & \multicolumn{3}{|c|}{ Área nativa } & \multicolumn{3}{|c|}{ Área reforestada } & \multicolumn{3}{|c|}{ Área degradada } \\
\hline & & ¿ & 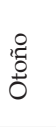 & 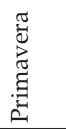 & 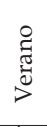 & 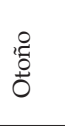 & 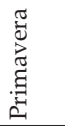 & 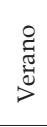 & 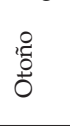 & 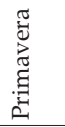 \\
\hline discomicetes & Discina sp. & & & & 1 & & & & & \\
\hline discomicetes & Hymenocyphus sp. & & & & & & 1 & & & \\
\hline discomicetes & Discomicete sp. 1 & 1 & & & & & & & & \\
\hline discomicetes & Discomicete sp. 2 & & & & 1 & & 7 & & & \\
\hline discomicetes & Discomicete sp. 3 & & & 7 & 5 & & 2 & & & \\
\hline discomicetes & Discomicete sp. 4 & & 1 & & 4 & & & & & \\
\hline discomicetes & Discomicete sp. 5 & & & & 1 & 1 & & & & \\
\hline discomicetes & Discomicete sp. 6 & & & & 1 & & & & & \\
\hline discomicetes & Discomicete sp. 7 & & & & & & & 3 & & \\
\hline discomicetes & Discomicete sp. 8 & & & & & & & 1 & & \\
\hline discomicetes & Discomicete sp. 9 & & 1 & & & & & & & \\
\hline discomicetes & Discomicete sp. 10 & & & 1 & & & 1 & & & \\
\hline discomicetes & Discomicete sp. 11 & & & 1 & & & 1 & & & \\
\hline discomicetes & Discomicete sp. 12 & & & & & & 1 & & & \\
\hline discomicetes & Discomicete sp. 13 & & & & & & & & & 1 \\
\hline discomicetes & Discomicete sp. 14 & & & & & & & & & 1 \\
\hline discomicetes & Discomicete sp. 15 & & & & & & & & & 1 \\
\hline otros ascomicetes & Claviceps purpurea (Fr.) Tul. & & & 1 & & & & & & \\
\hline otros ascomicetes & Cordyceps conf. militaris & & & & 1 & & & & & \\
\hline otros ascomicetes & Cordyceps sp. & & & & 1 & & & & & \\
\hline otros ascomicetes & Ascomicete sp. 1 & 1 & & & & & & & & \\
\hline otros ascomicetes & Ascomicete sp. 2 & 1 & & & & & & & & \\
\hline otros ascomicetes & Ascomicete sp 3 & & & & & & 1 & & & \\
\hline agaricoides & Coprinus sp. & & & & & & & 2 & & 6 \\
\hline agaricoides & Gymnopilus sp. & 2 & & & & & & & & \\
\hline agaricoides & Hygrocybe sp. & 4 & & & 1 & & & 1 & & \\
\hline agaricoides & Mycena sp. & 3 & & & 1 & & 1 & & & \\
\hline agaricoides & Psilocybe sp. & & & & & & & 6 & & \\
\hline agaricoides & Resupinatus sp. & & 3 & & & & & & & \\
\hline agaricoides & Agarical sp. 1 & & & & & & & 1 & 1 & \\
\hline agaricoides & Agarical sp. 2 & 7 & & & & & & & & \\
\hline agaricoides & Agarical sp. 3 & 2 & & & 3 & & & & & \\
\hline agaricoides & Agarical sp. 4 & & & & 1 & & & & & \\
\hline agaricoides & Agarical sp. 5 & & 1 & & & & & & & \\
\hline agaricoides & Agarical sp. 6 & 2 & & & 1 & & & & & \\
\hline agaricoides & Agarical sp. 7 & 1 & & & & & & & & \\
\hline agaricoides & Agarical sp, 8 & & 1 & & & & & 1 & & \\
\hline agaricoides & Agarical sp. 9 & 1 & & & & & & & & \\
\hline agaricoides & Agarical sp. 10 & 1 & & & & & & & & \\
\hline agaricoides & Agarical sp. 11 & 4 & & & & & & & & \\
\hline agaricoides & Agarical sp. 12 & 1 & & & & & & & & \\
\hline agaricoides & Agarical sp. 13 & & & 1 & & & & & & \\
\hline agaricoides & Agarical sp. 14 & & & 1 & & & & & & \\
\hline corticioides & $\begin{array}{l}\text { Aleurocystis gloeocystidiata } \\
\text { Rajchenb. \& Robledo }\end{array}$ & 12 & 29 & 12 & 7 & 14 & 23 & & & \\
\hline corticioides & Asterostroma conf ochroleucum & 1 & & & & & & & & \\
\hline corticioides & $\begin{array}{l}\text { Ceraceomyces eludens K.H. } \\
\text { Larss. }\end{array}$ & 1 & & & & & & & & \\
\hline
\end{tabular}


Tabla 3. Continuación.

Table 3. Continuation.

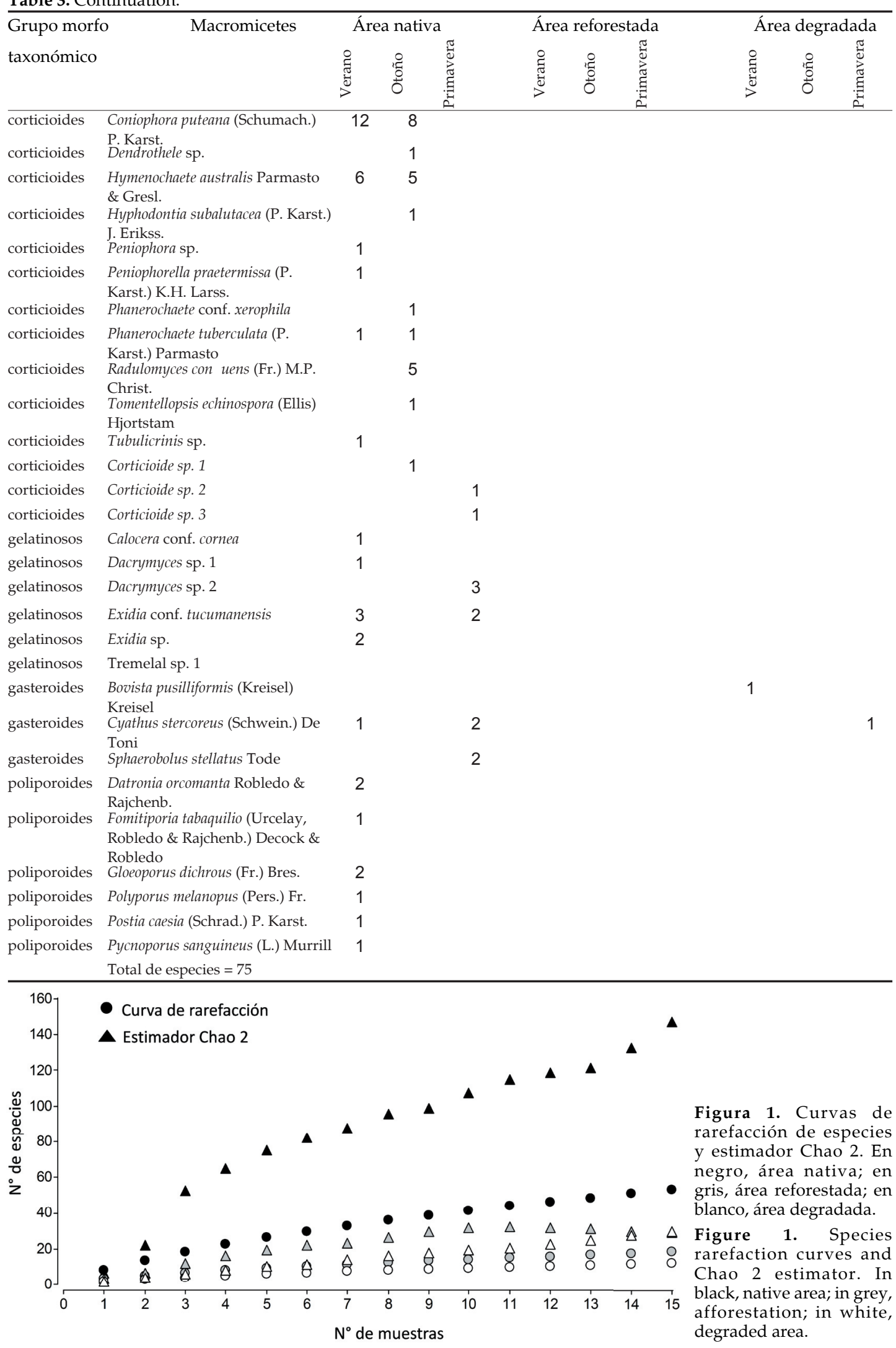



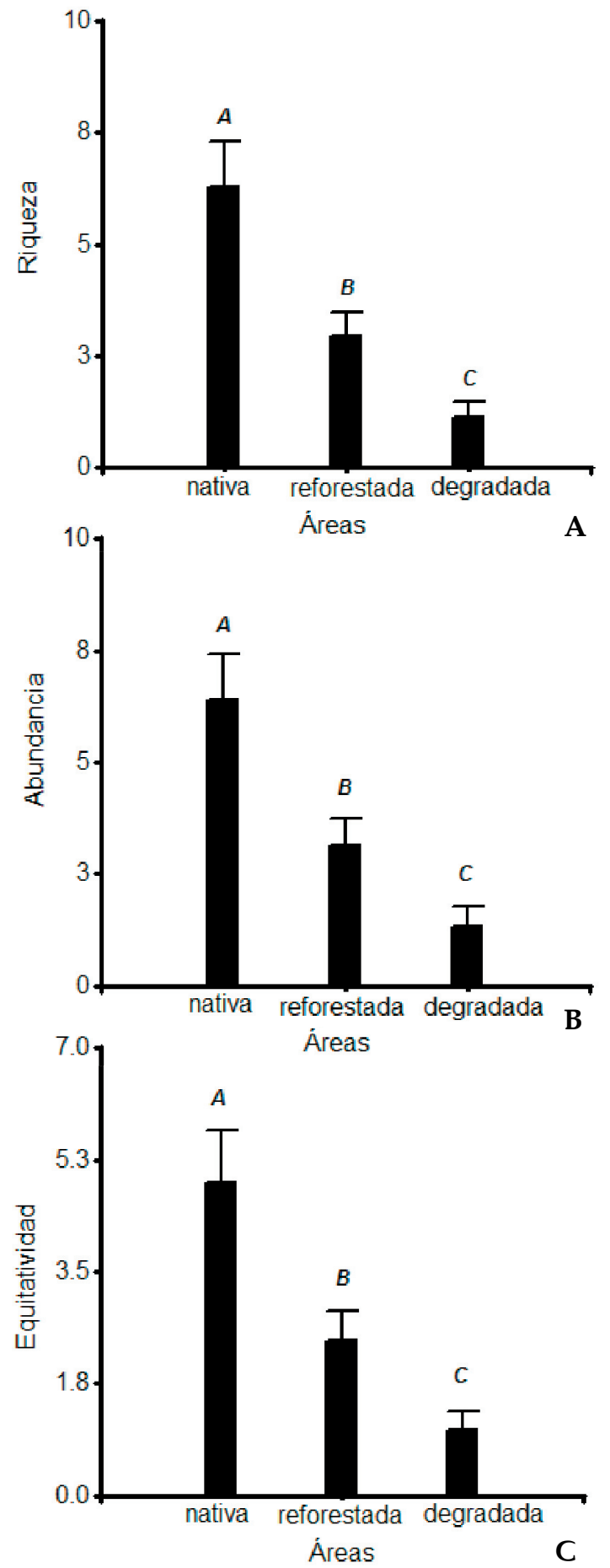

Figura 2. Diversidad fúngica promedio en las tres áreas de estudio (área nativa, área reforestada, área degradada) A. Riqueza de macromicetes (número promedio de especies). B. Abundancia de macromicetes (número promedio de macromicetes). C. Equitatividad de macromicetes (1/Índice de Simpson). Letras distintas indican diferencias significativas.

Figure 2. Mean fungal diversity in the three study areas (native area, afforestation, degraded area). A. Richness of macromicetes (mean species number). B. Abundance of macromicetes (mean macromicetes number). C. Evenness of macromicetes (1/Simpson's index). Different letters indicate significant differences. macromicetes. Dentro del Phylum Ascomycota, se registraron 17 especies de discomicetes y otras seis especies incluidas en el grupo "otros ascomicetes". Dentro del Phylum Basidiomycota, se encontraron 20 especies de hongos agaricoides, 17 de corticioides, 6 de gelatinosos, 3 de gasteroides y 6 de poliporoides (Tabla 3). En el AN se registraron 178 esporomas, correspondiente a 55 especies. En el AR se registraron 81, correspondientes a 18 especies, y en el AD, 27, correspondientes a 12 especies.

Las curvas de rarefacción muestran diferencias en la acumulación de especies entre las áreas. El AN alcanza los valores más altos, el AD los más bajos, mientras que el AR muestra valores intermedios pero próximos a los del AD. El estimador Chao 2 revela que los valores de riqueza estimados para AR y AD se encuentran próximos a los de la curva de rarefacción, mientras que la riqueza estimada en $\mathrm{AN}$ es más del doble de la observada (Figura 1). Se observaron diferencias significativas en la riqueza, abundancia y equitatividad de macromicetes entre las diferentes áreas $(P<0.001)$. La riqueza, la abundancia y la equitatividad fueron significativamente mayores en el AN, intermedias en el AR y menores en el AD (Figuras 2A-C). Además se observaron diferencias significativas entre estaciones para riqueza $(P=0.0001)$, abundancia $(P<0.0256)$ y equitatividad $(P<0.0001)$. En verano se observaron los valores significativamente más altos, mientras que en otoño, los más bajos. La riqueza relativa de especies de cada

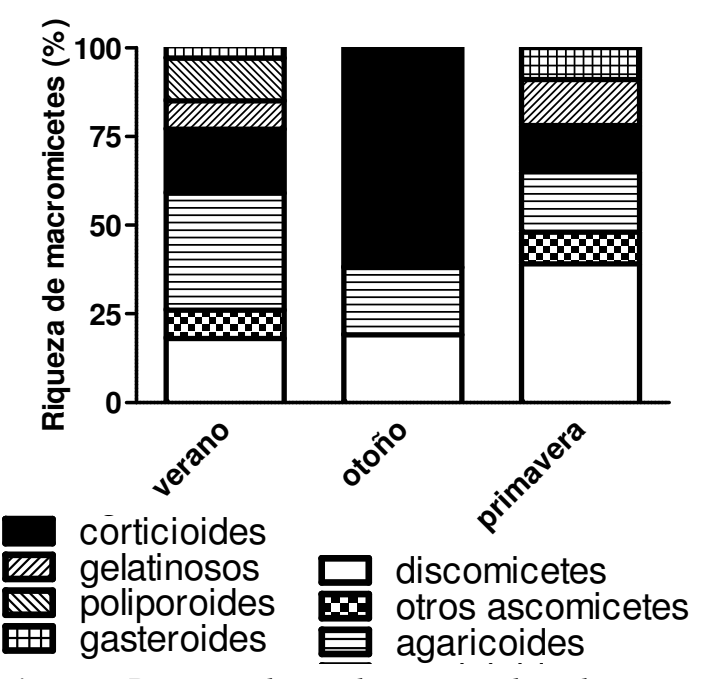

Figura 3. Riqueza relativa de especies de cada grupo morfo-taxonómico en tres estaciones.

Figure 3. Relative species richness in each morphotaxonomic group in three seasons. 

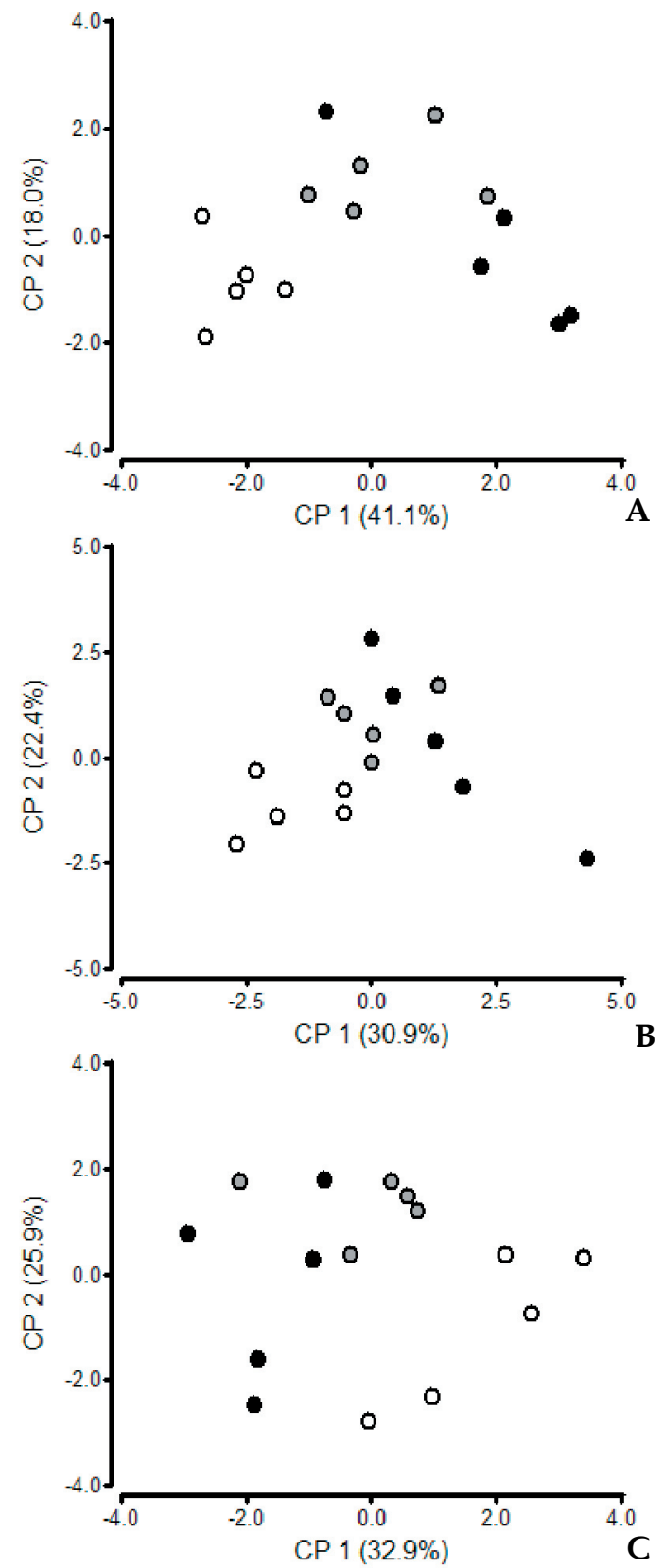

Figura 4. Análisis de Componentes Principales basado en variables estructurales de vegetación (roca, mantillo, árboles, suelo, herbáceas latifoliadas, arbustos, helechos, graminoides, briófitas y guano). A. Verano. B. Otoño. C. Primavera. En negro, área nativa; en gris, área reforestada; en blanco, área degradada.

Figure 4. Principal Components Analysis based on structural variables of vegetation (bare rock, litter, trees, bare soil, forbs, shrubs, ferns, graminoids, briophytes, and faeces). A. Summer. B. Autumn. C. Spring. In black, native area; in grey, afforestation; in white, disturbed area.

grupo morfo-taxonómico fue distinta (Figura 3). En verano se encontraron 51 especies y todos los grupos fueron registrados, aunque en diferentes porcentajes: los agaricoides constituyeron $33 \%$, los discomicetes y
Tabla 4. Contribución de las variables estructurales (autovalores) a los primeros dos ejes del Análisis de Componentes Principales correspondientes a las figuras 3A, B y C.

Tabla 4. Contribution of the structural variables (eigenvalues) to the first two axis of the Principal Components Analysis corresponding to figures 3A, B and $\mathrm{C}$.

\begin{tabular}{lcccccc}
\hline & \multicolumn{2}{c}{ Verano } & \multicolumn{2}{c}{ Otoño } & \multicolumn{2}{c}{ Primavera } \\
& Eje1 & Eje2 & Eje1 & Eje2 & Eje1 & Eje2 \\
\hline Roca & 0.03 & 0.35 & 0.28 & -0.35 & -0.17 & -0.43 \\
Mantillo & 0.28 & 0.32 & 0.46 & 0.04 & -0.39 & 0.25 \\
Árboles & 0.46 & -0.10 & 0.39 & 0.23 & -0.39 & 0.36 \\
Suelo & -0.31 & -0.10 & -0.22 & -0.41 & 0.08 & 0.02 \\
Herbáceas & -0.38 & -0.32 & -0.37 & -0.11 & 0.44 & -0.15 \\
latifoliadas & 0.36 & -0.24 & 0.06 & 0.47 & -0.33 & 0.11 \\
Arbustos & 0.39 & -0.32 & 0.27 & -0.03 & -0.35 & -0.27 \\
Helechos & -0.19 & 0.57 & 0.25 & 0.54 & 0.06 & 0.56 \\
Graminoides & -0.24 & 0.04 & 0.39 & -0.25 & -0.30 & -0.44 \\
Briófitas & -0.30 & -0.41 & -0.29 & -0.26 & 0.38 & -0.00 \\
Guano & -0.00
\end{tabular}

corticioides $18 \%$, los poliporoides $12 \%$, los hongos gelatinosos y "otros ascomicetes" $8 \%$ y los gasteroides sólo el 4\% (Figura 3). En esta estación, los agaricoides y corticioides fueron los más abundantes, constituyendo $35 \%$ y $34 \%$ de los registros, respectivamente, mientras que el resto de los grupos fueron menos abundantes $(14 \%$ para discomicetes, $6 \%$ para hongos gelatinosos y poliporoides, $3 \%$ para "otros ascomicetes" y $2 \%$ para gasteroides). En otoño se registraron 16 especies pertenecientes a 3 grupos: el 63\% correspondió a corticioides y el $19 \%$ a discomicetes y a agaricoides (Figura 3). La abundancia de corticioides fue del $89 \%$, mientras que la de agaricoides y discomicetes fue mucho menor ( $7 \%$ y $4 \%$, respectivamente). En primavera se registraron 23 especies y sólo

Tabla 5. Análisis de correlación entre variables fúngicas (riqueza, abundancia, equitatividad de macromicetes) y variables químicas del suelo (materia orgánica, nitrógeno total, nitrato, amonio, fósforo y $\mathrm{pH}$ ). ${ }^{*} P \leq 0.1,{ }^{* *} P \leq 0.05,{ }^{* * *} P \leq 0.01$

Table 5. Correlation analysis between fungal variables (richness, abundance and evenness of macromicetes) and soil chemical variables (organic matter, total nitrogen, nitrate, ammonium, phosphorus and $\mathrm{pH}$ ). ${ }^{*} P \leq 0.1,{ }^{* *} P \leq 0.05,{ }^{* * * P} \leq 0.01$

\begin{tabular}{lccc}
\hline & Riqueza & Abundancia & Equitatividad \\
\hline $\begin{array}{l}\text { Materia } \\
\text { orgánica (\%) }\end{array}$ & $0,31^{*}$ & $0,43^{* *}$ & 0,26 \\
$\begin{array}{l}\text { Nitrógeno } \\
\text { total (\%) }\end{array}$ & 0,25 & $0,39^{* *}$ & 0,21 \\
$\begin{array}{l}\text { Nitrato } \\
\text { (ppm) }\end{array}$ & 0,24 & 0,27 & 0,21 \\
$\begin{array}{l}\text { Amonio } \\
\text { (ppm) }\end{array}$ & 0,2 & 0,21 & 0,18 \\
$\begin{array}{l}\text { Fósforo } \\
\text { (ppm) }\end{array}$ & $0,61^{* * *}$ & $0,55^{* * *}$ & $0,59^{* * *}$ \\
pH & $0,48^{* * *}$ & $0,39^{* *}$ & $0,47^{* *}$ \\
\hline
\end{tabular}


el grupo de los poliporoides no fue registrado (Figura 3). El 39\% de las especies encontradas correspondió a discomicetes, $17 \%$ a agaricales, $13 \%$ a corticioides y hongos gelatinosos y $9 \%$ a "otros ascomicetes" y gasteroides. A pesar de que la mayoría de los grupos estuvieron presentes en esta estación, los corticioides fueron los más abundantes (44\%), los discomicetes y agaricoides intermedios (29\% y $12 \%$, respectivamente), mientras que el resto de los grupos fueron poco abundantes $(7 \%, 6 \%$ y $2 \%$ para hongos gelatinosos, gasteroides y "otros ascomicetes", respectivamente).

En el análisis de componentes principales, las parcelas de $\mathrm{AN}$ se caracterizaron en todos los casos por una alta cobertura arbórea y, en menor medida, por arbustos, helechos, mantillo y briófitas. Las parcelas del AR se caracterizaron por una importante cobertura de graminoides y las del AD por herbáceas latifoliadas (Tablas 1 y 4, Figura 4). Estas tendencias fueron más marcadas en verano, donde el AN se separó del AD y el AR presentó una situación intermedia (Figura 4A). En otoño y en primavera, el AD se encontró nuevamente en un extremo del espacio multivariado; sin embargo, no hubo aquí una marcada separación entre el AN y el AR (Figuras 4B y C).

El eje 1 del ACP se correlacionó significativamente con la riqueza, la abundancia y la equitatividad, tanto en verano $\left(r^{2}=0.86, P<0.0001 ; r^{2}=0.82, P=0.0002 y^{2}=0.81\right.$, $P=0.0002$, respectivamente) como en otoño $\left(r^{2}=0.76, P=0.0009 ; r^{2}=0.80, P=0.0004 y^{2} r^{2}=0.79\right.$, $P=0.0004$, respectivamente). En primavera no se encontraron correlaciones significativas. Las tres variables fúngicas fueron máximas a mayor cobertura arbórea. Además, en verano se relacionaron con mayores coberturas de arbustos y helechos, y en otoño con mayores coberturas de mantillo y briófitas (Tablas 1 y 4). A su vez, la relación fue negativa con la cobertura de herbáceas latifoliadas.

La tres variables fúngicas se correlacionaron de forma positiva principalmente con el contenido de fósforo y, en menor medida, con el $\mathrm{pH}$. Además, la riqueza y la abundancia lo hicieron con el contenido de materia orgánica. La abundancia también se correlacionó positivamente con el contenido de nitrógeno total (Tabla 5).

\section{DISCUSIÓN}

En este estudio observamos que la composición de las comunidades de hongos varía entre las distintas situaciones de bosque consideradas. El estimador de riqueza Chao 2 revela que los valores del AR y del AD están próximos a los observados en las curvas de rarefacción, mientras que la riqueza estimada para el AN es bastante más alta que la observada. Estos datos sugieren que, a pesar del esfuerzo de muestreo, se deberían realizar más muestreos para abarcar la riqueza del AN. Por lo tanto, las diferencias entre AN y las otras dos situaciones deben considerarse conservadoras.

Los resultados de este estudio sugieren que la reforestación es efectiva para incrementar el número de especies de macromicetes al compararla con un área degradada. No obstante, los valores de las variables fúngicas encontrados luego de doce años desde el inicio de la reforestación son notablemente menores que los del AN.

La diversidad de macromicetes determinada a partir del registro de esporomas, práctica frecuente para estos organismos (e.g., Bader et al. 1995; Heilmann-Clausen \& Christensen 2003; Urcelay \& Robledo 2004; Gates et al. 2005; Brown et al. 2006; Lindner et al. 2006; Kujawa \& Kujawa 2008; Blaser et al. 2013), no incluye especies cuya fase vegetativa está presente en el sustrato pero no fructifican. Si bien esto constituye una limitación de estos estudios, cabe destacar que más allá de algunas diferencias en la detección de algunas especies, el uso de herramientas moleculares para la identificación de micelio vegetativo en el sustrato arroja conclusiones similares a las del análisis de las comunidades de hongos a partir de la ocurrencia de esporomas, al menos en hongos de la madera (Ovaskainen et al. 2013).

La disponibilidad de recursos, así como su variación en el espacio y en el tiempo, constituyen los principales factores estructuradores de las comunidades fúngicas a escala local, e influyen sobre su composición taxonómica y funcional (Ponge 2005). En este sentido, la estructura de las comunidades vegetales es uno de los factores determinantes de las comunidades fúngicas. En función de la cobertura vegetal dada por distintos grupos de plantas, en el ACP, las parcelas del AN se ubicaron en un extremo del espacio multivariado, mientras que las del AD lo hicieron en el otro. A su vez las parcelas del AR mostraron una posición intermedia (Figuras 4A, B y C). En concordancia, la 
riqueza y abundancia de hongos fue mayor en el AN que posee, en comparación con las otras situaciones, la mayor complejidad estructural y biomasa vegetal (Tablas 1 y 4). Esto es consistente con los resultados de Gates et al. (2005), quienes observaron que la riqueza de macromicetes en bosques maduros de Eucalyptus obliqua en Tasmania era el doble que en forestaciones de pocos años.

Tanto la cantidad como la variedad de madera son factores estructuradores clave de la riqueza de especies de hongos degradadores de la madera (Blaser et al. 2013). Estudios previos han mostrado que la cantidad de troncos y la variedad de diámetros influyen de manera positiva sobre la diversidad (Heilmann-Clausen \& Christensen 2003; Boddy \& Heilmann-Clausen 2008; Yamashita et al. 2008; Robledo \& Renison 2010) y sobre la producción de esporomas de hongos degradadores de la madera (Urcelay \& Robledo 2009). El hecho de que los hongos degradadores de la madera, incluidos aquí en "poliporoides" y "corticioides", hayan sido observados exclusivamente en el AN pone de manifiesto que el AR todavía no presenta ramas o troncos de diámetro suficiente para albergar este grupo funcional de hongos. Esto explica parte de las diferencias que se observan entre el AR y el AN.

Por otro lado, existen otros grupos de hongos que se ven favorecidos por las características de los bosques más jóvenes (Kujawa \& Kujawa 2008). Tal el caso de los discomicetes, que en el presente estudio se observaron principalmente en el AR (Tabla 3), creciendo sobre gramíneas formadoras de matas. El AR se caracteriza por una gran cobertura de este grupo de plantas y la mitad de las especies fúngicas encontradas en el área reforestada correspondieron a discomicetes (Tabla 3).

En forestaciones con diversas especies de árboles se ha observado que la proporción de herbáceas latifoliadas se relaciona de manera negativa con la riqueza de macromicetes (Kujawa \& Kujawa 2008). Además, las comunidades vegetales con menor cobertura de la canopia albergan un número menor de especies fúngicas debido a mayor temperatura y menor humedad (Brown et al. 2006). En este sentido, el presente estudio muestra que la diversidad fúngica se relacionó positivamente con la cobertura arbórea y, en menor medida, con la arbustiva, helechos, briófitas y mantillo.
Junto a la vegetación, las variables químicas del suelo constituyen otro de los importantes factores estructuradores de las comunidades de hongos a escala del ecosistema (Ettema \& Wardle 2002). Las variables fúngicas se correlacionaron positiva y significativamente con el fósforo y, en menor medida, con el pH, con el contenido de materia orgánica y con el nitrógeno total. Estos resultados coinciden en parte con estudios previos que muestran que los nutrientes, en particular el fósforo (e.g., Lauber et al. 2008), y en menor medida el pH (e.g., Rousk et al. 2010), pueden ser predictores de los cambios en las comunidades de hongos. En conjunto, estos resultados sugieren que la complejidad estructural de las comunidades vegetales y variables químicas del suelo tales como el fósforo, $\mathrm{pH}$, materia orgánica y nitrógeno total, serían los principales factores a través de los cuales la restauración del bosque promueve una mayor diversidad de hongos.

El presente estudio involucró muestreos en tres estaciones del año con el fin de abarcar la variabilidad fenológica en la producción de esporomas, ya que la ocurrencia de los mismos y el ensamble de especies se relacionan con la estación y el clima (como se ha mostrado en otros trabajos [e.g., Gates et al. 2005]). La riqueza relativa de especies de cada grupo varió entre estaciones. La riqueza, la abundancia y la equitatividad de macromicetes fueron mayores en verano, única estación en que todos los grupos morfotaxonómicos fueron registrados (Figura 3). Un patrón similar se observó en la disponibilidad de nutrientes (fósforo, nitrato y amonio) que mostraron valores más altos en verano, aunque fueron similares entre las situaciones de bosque dentro de las estaciones.

A pesardequesuele ser una realidad frecuente en los estudios de restauración ecológica (Ruiz-Jaen \& Aide 2005), es importante señalar que las situaciones de bosque de este estudio no se repiten en el paisaje. Esto se debe a que el AR es una situación única en la región lo que constituye una limitación insalvable. No obstante, esta reforestación es la única que cuenta con árboles de más de $2 \mathrm{~m}$ de altura y, por ende, con la presencia de detritos leñosos. Por ello, consideramos que los resultados son particularmente relevantes en un marco descriptivo, y deben ser considerados como una primera aproximación que establece las bases para estudios posteriores cuando las situaciones descriptas se repitan en el tiempo 
y el espacio. La generalización de estos resultados dependerá de tales estudios.

\section{CONCLUSIONES}

Los resultados presentados aquí muestran que 12 años después de que un área degradada fuera reforestada con plantines de $P$. australis, la diversidad fúngica es mayor a la observada en un área degradada de referencia. No obstante, dicha diversidad está lejos de alcanzar los niveles observados en el bosque maduro. Es importante destacar que la diversidad de hongos se relacionó en buena medida a la complejidad estructural de la vegetación. Debido a que dicha complejidad suele aumentar con la edad del bosque, es posible especular que la diversidad de hongos en la reforestación seguirá incrementando sus niveles con el transcurrir de los años. Si bien esta es una primera aproximación a la utilización de la diversidad fúngica para evaluar el éxito de una reforestación con especies nativas y presenta limitaciones, es posible postular que los hongos serían bioindicadores sensibles a la degradación y posterior restauración de los bosques. Esto pone en relieve su importancia en la evaluación integral de la restauración de los ecosistemas.

Agradecimientos: Los autores agradecen a The Rufford Small Grant for Nature Conservation y a FONCyT por el financiamiento para realizar el trabajo. A D. Renison por incentivar y proveer facilidades para el desarrollo de este estudio y a S. Pérez Gorjón por la identificación de los hongos corticioides. G. Robledo y C. Urcelay son investigadores del CONICET y agradecen el apoyo del Consejo Nacional de Investigaciones Científicas y Técnicas (CONICET) y la Universidad Nacional de Córdoba por haber provisto las facilidades para realizar este trabajo. Los autores también agradecen los comentarios y sugerencias de varios revisores anónimos.

\section{BiBLIOGRAFÍA}

BADER, P; S JANSSON \& BG Jonsson. 1995. Wood-inhabiting fungi and substratum decline in selectively logged boreal spruce forests. Biol. Conserv., 72(3):355-362.

Blaser, S; D Prati; B Senn-Irlet \& M Fischer. 2013. Effects of forest management on the diversity of deadwoodinhabiting fungi in Central European forests. Forest Ecol. Manag., 304:42-48.10.1016/j.foreco.2013.04.043.

Boddy, L \& J Heilmann-Clausen. 2008. Basidiomycete community development in temperate angiosperm wood (capítulo 12). Pp. 211-237 en: BoDDY, L; JC FrANKLAND \& P VAN WEST (eds.). Ecology of saprotrophic basidiomycetes. 1a. ed. Academic Press. Aberdeen, UK. Pp. 372.

BRown, N; S Bhagwat \& S WATKINSON. 2006. Macrofungal diversity in fragmented and disturbed forests of the Western Ghats of India. J. Appl. Ecol., 43(1):11-17.

CABido, M. 1985. Las comunidades vegetales de la Pampa de Achala, Sierras de Córdoba, Argentina. Doc.
Phytosociol., 9:431-43.

Cabido, M; G Funes; E Pucheta; F Vendramini \& S Díaz. 1998. A chronological analysis of the mountains from Central Argentina. Is all what we call Sierra Chaco really Chaco? Contribution to the study of the flora and vegetation of the Chaco. Candollea, 53:321-331.

CABIDO, M; A Antón; M CABrera; AM Cingolani; I Di TADA; ET AL. 2003. Línea de base y programa de monitoreo de la biodiversidad del Parque Nacional Quebrada del Condorito y la Reserva Hídrica Provincial Pampa de Achala. Internal Report.

Cingolani, AM; D Renison; PA Tecco; DE Gurvich \& M CABIDO. 2008. Predicting cover types in a mountain range with long evolutionary grazing history: a GIS approach. J. Biogeogr., 35(3):538-551.

Cingolani, AM; D Renison; M ZaK \& M Cabido. 2004. Mapping vegetation in a heterogeneous mountain rangeland using Landsat data: an alternative method to define and classify land-cover units. Remote Sens. Environ., 92(1):84-97.

Chapman, M \& A Underwood. 2000. The need for a practical scientific protocol to measure successful restoration. Wetlands, 19(1):28-49.

CHAZDON, R. 2008. Beyond deforestation: restoring forests and ecosystem services on degraded lands. Science, 320(5882):1458-1460.

Colladon, L. 2000. Anuario Pluviométrico 1992-2000. Cuenca del Río San Antonio. Sistema del Río Suquía-Provincia de Córdoba. Instituto Nacional del Agua y del Ambiente (INAA) y Centro de Investigaciones de la Región Semiárida (CIRSA).

Colwell, RK. 2009. EstimateS: Statistical estimation of species richness and shared species from samples. http: //viceroy.eeb.uconn.edu/estimates/

Cromack Jr, K \& BA Caldwell. 1992. The role of fungi in litter decomposition and nutrient cycling (capítulo 33). Pp. 653-668 en: CARRoll, GC \& DT WickLow (eds.). The fungal community. Its organization and role in the ecosystem. 2a. ed. Marcel Dekker, Inc. Nueva York, EEUU. Pp. 976 pp.

Di Rienzo, JA; F CASANOVES; MG BalZarini; L GonZÁlez; M TABLADA; ET AL. 2013. InfoStat. http://www.infostat.com.ar

ENRICO, L; G FunES \& M CABIDO. 2004. Regeneration of Polylepis australis BITT. in the mountains of central Argentina. Forest Ecol. Manag., 190:301-309.

EtTema, CH \& DA WardLe. 2002. Spatial soil ecology. Trends in Ecology \& Evolution, 17:177-183.

FJeldsÅ, J \& M Kessler. 1996. Conserving the biological diversity of Polylepis woodlands of the highland of Peru and Bolivia. A Contribution to Sustainable Natural Resource Management in the Andes. NORDECO. Copenhague, Denmark. Pp. 250.

Gates, GM; DA RatKowsky \& SJ Grove. 2005. A comparison of macrofungi in young silvicultural regeneration and mature forest at the Warra LTER Site in the southern forests of Tasmania. Tasforests, 16:127-152.

Heilmann-Clausen, J \& M Christensen. 2003. Fungal diversity on decaying beech logs -implications for sustainable forestry. Biodivers. Conserv., 12(5):953-973.

HeRnándeZ CAFFot, ML; G RoBLEDO \& LS DomíngueZ. 2013. Gasteroid mycobiota (Basidiomycota) from Polylepis australis woodlands of central Argentina. Mycotaxon, 123:491-502.

HoBBS, RJ. 2007. Setting effective and realistic restoration goals: key directions for research. Restor. Ecol., 15(2): 354-357. 
HobBS, RJ; S ARICO; J ARONSON; JS BARON; P BRIDGEWATER; ET AL. 2006. Novel ecosystems: theoretical and management aspects of the new ecological world order. Global Ecol. Biogeogr., 15(1):1-7.

Kent, M \& P COKER. 1992. Vegetation description and analysis: a practical approach. Boca Ratón:CRC press. Florida, EEUU. Pp. 363.

KujaWA, A \& K KuJaWA. 2008. Effect of young midfield shelterbelts development on species richness of macrofungi communities and their functional structure. Pol. J. Ecol., 56(1):45-56.

Lauber, CL; MS Strickland; MA Bradford \& N Fierer. 2008. The influence of soil properties on the structure of bacterial and fungal communities across land-use types. Soil Biol. Biochem., 40:2407-2415.

LindNER, D; HH BuRdsall JR \& GR Stanosz. 2006. Species diversity of polyporoid and corticioid fungi in northern hardwood forests with differing management histories. Mycologia, 98(2):195-217.

Moore, D; AC Gange; EG Gange \& L Boddy. 2008. Fruit bodies: their production and development in relation to environment (capítulo 5). Pp. 94 en: BodDY, L; JC FRANKLAND \& P VAN WEST (eds.). Ecology of saprotrophic basidiomycetes. 1a. ed. Academic Press. Aberdeen, UK. Pp. 372.

Oria de Rueda, JA; M Hernández Rodríguez; P MartínPinto; V Pando \& J Olaizola. 2010. Could artificial reforestations provide as much production and diversity of fungal species as natural forest stands in marginal Mediterranean areas? Forest Ecol. Manag., 260:171-180.

Ovaskainen, O; D Schigel; H Ali-Kovero; P Auvinen; L Paulin; ET AL. 2013. Combining high-throughput sequencing with fruit body surveys reveals contrasting life-history strategies in fungi. The ISME Journal, 7(9): 1696:1709.

PonGE, JF. 2005. Fungal communities: relation to resource succession (capítulo 8). Pp 169-180 en: Dighton, J; JF WhITE \& POUdEMANs. (eds.). The fungal community. Its organization and role in the ecosystem. 3a ed. Boca Ratón:CRC press, Taylor \& Francis Group. NY, EEUU. Pp. 936.

Renison, D; I Hensen; R SuÁrez; AM Cingolani; P Marcora; ET AL. 2010. Soil conservation in Polylepis mountain forests of Central Argentina: Is livestock reducing our natural capital? Austral Ecology, 35:435-443.

Renison, D; GE CuYcKens; S PACHeCo; GF GuZmán; HR GRAU; ET AL. 2013. Distribución y estado de conservación de las poblaciones de árboles y arbustos del género Polylepis (Rosaceae) en las montañas de Argentina. Ecología Austral, 23(1):27-36.

Renison, D; I Hensen; R Suárez \& AM Cingolani. 2006. Cover and growth habit of Polylepis woodlands and shrublands in the mountains of central Argentina: human or environmental influence? J. Biogeogr., 33(5): 876-887.

Robledo, G; C URCELAY; LS DOMÍNGUEZ \& M RAJCHENBERG. 2006. Taxonomy, ecology and biogeography of polypores (Badiomycetes) from Argentinian Polylepis woodlands. Can. J. Bot., 84(10):1561-1572.

Robledo, G \& D ReNISON. 2010. Wood-decaying polypores in the mountains of central Argentina in relation to Polylepis forest structure and altitude. Fungal Ecol., 3(3): 178-184.

RUIZ-JAEN, MC \& TM AIDE. 2005. Restoration success: how is it being measured? Restor. Ecol., 13(3):569-577.

RousK, J; E BÅÅth; PC BROOKes; CL LaUber; C LOZUPONE; ET AL. 2010. Soil bacterial and fungal communities across a $\mathrm{pH}$ gradient in an arable soil. ISME Journal, 4: 1340-1351.

SER (Society for Ecological Restoration Science and Policy Working Group). 2002. http://www.ser.org/

SPARKS, DL; AL PAGE; PA HelmKe; RH LOEPPERT; PN SOLtANPOUR; ET AL. (eds.). 1996. Methods of soil analysis. Chemical methods (Part 3). Soil Science Society of America book series. Madison, EEUU. Pp. 1390.

SwIFT, MJ. 2005. Human impacts on biodiversity and ecosystem services: an overview. (capítulo 31). Pp. 627641 en: Dighton, J; JF White \& P Oudemans. (eds.). The fungal community. Its organization and role in the ecosystem. 3a ed. Boca Raton: CRC press, Taylor \& Francis Group. NY, EEUU. Pp. 936.

Torres, RC; D Renison; I Hensen; R SuÁrez \& L Enrico. 2008. Polylepis australis regeneration niche in relation to seed dispersal, site characteristics and livestock density. Forest Ecol. Manag., 254(2):255-260.

Unterseher, M; M SChNitTler; C Dormann \& A SicKert. 2008. Application of species richness estimators for the assessment of fungal diversity. Microbiology Letters, 282(2):205-213.

Urcelay, C \& G Robledo. 2004. Community structure of polypores (Basidiomycota) in Andean Alder wood in Argentina: functional groups among wood-decay fungi? Austral Ecology, 29:471-476.

Urcelay, C \& G Robledo. 2009. Positive relationship between wood size and basidiocarp production of polypore fungi in Alnus acuminata forest. Fungal Ecol., 2(3):135-139.

VON MÜller, AR; AM Cingolani; MV Vaieretti \& D RENISON. 2012. Estimación de carga bovina localizada a partir de frecuencia de deposiciones en un pastizal de montaña. Ecología Austral, 22:178-187.

Wilkins, S; D KeITH \& P Adam. 2003. Measuring success: evaluating the restoration of a grassy Eucalypt woodland on the Cumberland Plain, Sydney, Australia. Restor Ecol., 11(4):489-503.

Yamashita, S; T Hattori; K Momose; M NaKagawa; M Aiba; ET AL. 2008. Effects of forest use on Aphyllophoraceous fungal community structure. Biotropica, 40(3):354-362.

ZAR, JH. 1999. Biostatistical Analysis. 4th ed. Prentice Hall Inc. Upper Saddle River, NJ, EEUU. 